\title{
The competition number of a generalized line graph is at most two
}

\author{
Boram Park ${ }^{1 \dagger}$ and Yoshio Sano ${ }^{2 \ddagger}$ \\ ${ }^{1}$ DIMACS, Rutgers University, Piscataway, NJ 08854, United States \\ ${ }^{2}$ National Institute of Informatics, Tokyo 101-8430, Japan \\ received $13^{\text {th }}$ October 2011, accepted $16^{\text {th }}$ July 2012.
}

In 1982, Opsut showed that the competition number of a line graph is at most two and gave a necessary and sufficient condition for the competition number of a line graph being one. In this paper, we generalize this result to the competition numbers of generalized line graphs, that is, we show that the competition number of a generalized line graph is at most two, and give necessary conditions and sufficient conditions for the competition number of a generalized line graph being one.

Keywords: competition graph; competition number; generalized line graph

\section{Introduction}

The notion of a competition graph was introduced by Cohen [1] as a means of determining the smallest dimension of ecological phase space. The competition graph $C(D)$ of a digraph $D$ is a graph which has the same vertex set as $D$ and an edge between two distinct vertices $u$ and $v$ if and only if there exists a vertex $x$ in $D$ such that $(u, x)$ and $(v, x)$ are arcs of $D$. Roberts [12] observed that any graph $G$ together with sufficiently many isolated vertices is the competition graph of an acyclic digraph. The competition number $k(G)$ of a graph $G$ is defined to be the smallest nonnegative integer $k$ such that $G$ together with $k$ isolated vertices added is the competition graph of an acyclic digraph. It is not easy in general to compute $k(G)$ for an arbitrary graph $G$, since Opsut [8] showed that the computation of the competition number of a graph is an NP-hard problem. It has been one of the important research problems in the study of competition graphs to compute the exact values of the competition numbers of various graphs. For some special graph families, we have explicit formulae for computing competition numbers: If $G$ is a chordal graph without isolated vertices, then $k(G)=1$; If $G$ is a nontrivial triangle-free connected graph then $k(G)=|E(G)|-|V(G)|+2$ ([12]). For more recent results on graphs whose competition numbers are calculated exactly, see $[3,4,5,6,7,9,10,11,13]$.

\footnotetext{
${ }^{\dagger}$ Email: boramp@dimacs.rutgers.edu; This work was supported by National Research Foundation of Korea Grant funded by the Korean Government, the Ministry of Education, Science and Technology (NRF-2011-357-C00004).

${ }^{\ddagger}$ Email: sano@nii.ac.jp; The author was supported by JSPS Research Fellowships for Young Scientists. 
The line graph $L(H)$ of a graph $H$ is the simple graph defined by $V(L(H))=E(H)$ and $e e^{\prime} \in$ $E(L(H))$ if and only if $e$ and $e^{\prime}$ have a vertex in common and $e \neq e^{\prime}$. A graph $G$ is called a line graph if there exists a graph $H$ such that $G$ is isomorphic to the line graph of $H$. A clique $S$ of a graph $G$ is a set of vertices of $G$ such that the subgraph induced by $S$ is a complete graph (the empty set is also considered a clique). A vertex $v$ in a graph $G$ is called simplicial if the neighborhood of $v$ in $G$ is a clique of $G$. In 1982, Opsut [8] showed the following theorem.

Theorem 1.1 ([8]) For a line graph $G, k(G) \leq 2$ and the equality holds if and only if $G$ has no simplicial vertex.

In this paper, we investigate the competition number of a generalized line graph which was introduced by Hoffman [2] in 1970. For a positive integer $m$, the cocktail party graph $C P(m)$ is the complete multipartite graph with $m$ partite sets all of which have the size two:

$$
\begin{aligned}
V(C P(m))= & \bigcup_{l \in[m]}\left\{x_{l}, y_{l}\right\} \\
E(C P(m))= & \left\{x_{i} x_{j} \mid i, j \in[m], i<j\right\} \cup\left\{y_{i} y_{j} \mid i, j \in[m], i<j\right\} \\
& \cup\left\{x_{i} y_{j} \mid i, j \in[m], i \neq j\right\}
\end{aligned}
$$

where $[m]$ denotes the $m$-set $\{1,2, \ldots, m\}$. Note that $C P(1)$ is the graph with two vertices and no edge. A vertex-weighted graph $(H, f)$ is a pair of a graph $H$ and a non-negative integer-valued function $f: V(H) \rightarrow \mathbb{Z}_{\geq 0}$ on the vertex set of $H$. The generalized line graph $L(H, f)$ of a vertex-weighted graph $(H, f)$ is the graph obtained from the disjoint union of the line graph $L(H)$ of the graph $H$ and the cocktail party graphs $Q_{v}:=C P(f(v))$ where $v \in V(H)$ with $f(v)>0$ by adding edges between all the vertices in $Q_{v}$ and $e \in V(L(H))$ such that $e$ is incident to $v$ in $H$ (see Figure 1). For a graph $H$, if $f$ is a zero function (i.e. $f(v)=0$ for any $v \in V(H)$ ), then the generalized line graph of $(H, f)$ is the same as the line graph of $H$. A graph $G$ is called a generalized line graph if there exists a vertex-weighted graph $(H, f)$ such that $G$ is isomorphic to the generalized line graph of $(H, f)$.

In this paper, we show the following result.

Theorem 1.2 The competition number of a generalized line graph is at most two.

This paper is organized as follows. Section 2 is the main part of this paper. Subsection 2.1 gives some observations on the competition graphs of acyclic digraphs which will be used in this paper. Subsection 2.2 shows that the competition number of a generalized line graph is at most two. In Subsection 2.3, we investigate generalized line graphs whose competition numbers are one, and give some sufficient conditions and necessary conditions. Section 3 gives some concluding remarks.

\section{Main Results}

\subsection{Preliminaries}

For a digraph $D$ and a vertex $v$ of $D, N_{D}^{+}(v)$ and $N_{D}^{-}(v)$ denotes the out-neighborhood and the inneighborhood of $v$, respectively, i.e., $N_{D}^{+}(v):=\{x \in V(D) \mid(v, x) \in A(D)\}$ and $N_{D}^{-}(v):=\{x \in$ $V(D) \mid(x, v) \in A(D)\}$. A digraph is said to be acyclic if it contains no directed cycles. An ordering $v_{1}, \ldots, v_{|V(D)|}$ of the vertices of a digraph $D$ is called an acyclic ordering of $D$ if $\left(v_{i}, v_{j}\right) \in A(D)$ implies $i<j$. It is well-known that a digraph is acyclic if and only if it has an acyclic ordering. 


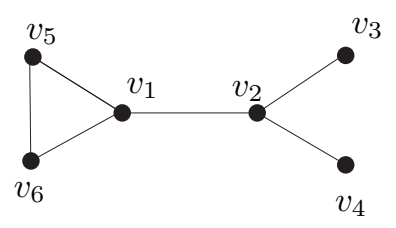

$H$

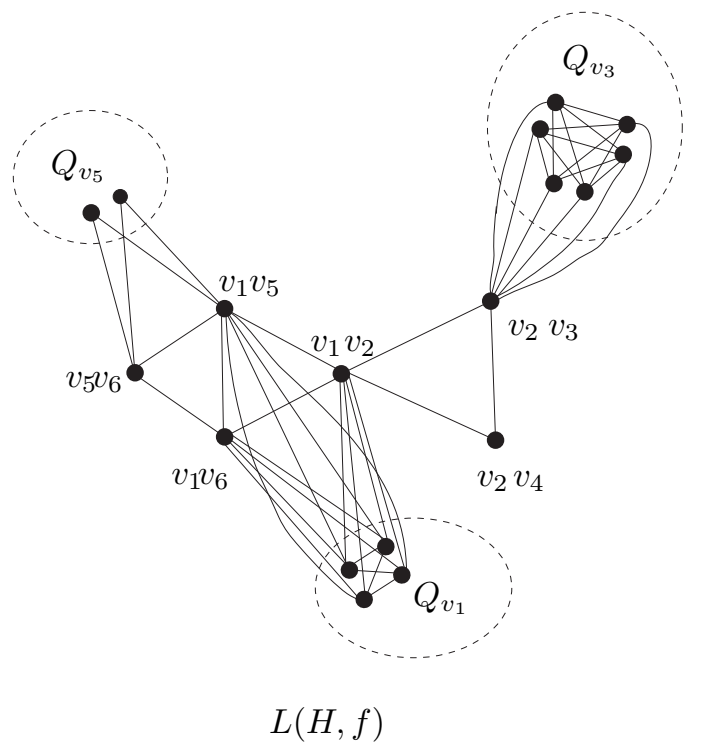

Fig. 1: A vertex-weighted graph $(H, f)$, where $f: V(H) \rightarrow \mathbb{Z}_{\geq 0}$ is defined by $f\left(v_{1}\right)=2, f\left(v_{3}\right)=3, f\left(v_{5}\right)=1$, $f\left(v_{2}\right)=f\left(v_{4}\right)=f\left(v_{6}\right)=0$, and its generalized line graph $L(H, f)$

For a clique $S$ of a graph $G$ and an edge $e$ of $G$, we say that $e$ is covered by $S$ if both of the endvertices of $e$ are contained in $S$. An edge clique cover of a graph $G$ is a family of cliques of $G$ such that each edge of $G$ is covered by some clique in the family. The edge clique cover number $\theta_{E}(G)$ of a graph $G$ is the minimum size of an edge clique cover of $G$. A vertex clique cover of a graph $G$ is a family of cliques of $G$ such that each vertex of $G$ is contained in some clique in the family. The vertex clique cover number $\theta_{V}(G)$ of a graph $G$ is the minimum size of a vertex clique cover of $G$. For a graph $G$ and a vertex $v$ of $G, \theta_{V}\left(N_{G}(v)\right)$ is the vertex clique cover number of the subgraph of $G$ induced by the neighbors of $v$ in $G$. Opsut [8] showed the following lower bound for the competition number of a graph (see also [14] for a generalization of this inequality).

Proposition 2.1 ([8]) For any graph $G, k(G) \geq \min _{v \in V(G)} \theta_{V}\left(N_{G}(v)\right)$.

For a positive integer $k$, we denote by $I_{k}$ the edgeless graph on $k$ vertices, i.e., the graph with $k$ vertices and no edges. The following lemma which comes from an easy observation is elementary but useful.

Lemma 2.2 Let $G$ be a graph with at least two vertices and let $k$ be an integer such that $k \geq k(G)$. Then there exists an acyclic digraph $D$ such that

(a) $C(D)=G \cup I_{k}$,

(b) $D$ has an acyclic ordering $v_{1}, \ldots, v_{|V(G)|}, v_{|V(G)|+1}, \ldots, v_{|V(G)|+k}$, where $V(G)=\left\{v_{1}, \ldots\right.$, $\left.v_{|V(G)|}\right\}$ and $V\left(I_{k}\right)=\left\{v_{|V(G)|+1}, \ldots, v_{|V(G)|+k}\right\}$, and

(c) $N_{D}^{-}\left(v_{1}\right)=N_{D}^{-}\left(v_{2}\right)=\emptyset$. 


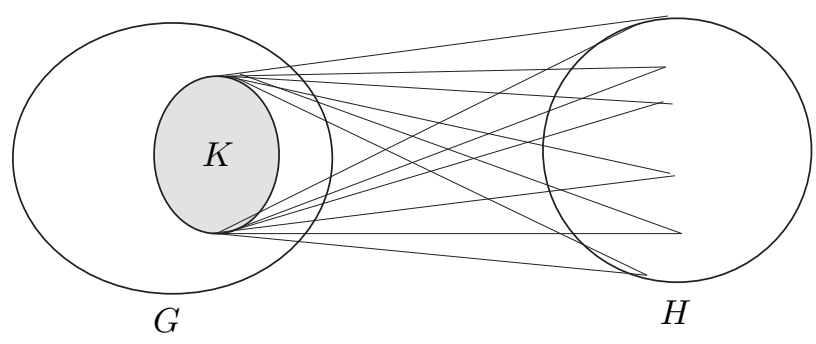

Fig. 2: $G \ltimes_{K} H$

Proof: By the definition of the competition number of a graph, there exists an acyclic digraph $D_{1}$ satisfying (a). Let $D_{2}$ be the digraph obtained from $D_{1}$ by deleting all the arcs outgoing from any vertices in $I_{k}$. Then we can check that $D_{2}$ is an acyclic digraph satisfying (a) and (b). Since there is no arc outgoing from any vertex in $I_{k}$, there is an acyclic ordering $v_{1}, v_{2}, \ldots, v_{|V(G)|}, v_{|V(G)|+1}, \ldots, v_{|V(G)|+k}$ of $D_{2}$ such that $V(G)=\left\{v_{1}, v_{2}, \ldots, v_{|V(G)|}\right\}$ and $V\left(I_{k}\right)=\left\{v_{|V(G)|+1}, \ldots, v_{|V(G)|+k}\right\}$. By the definition of an acyclic ordering of a digraph, it holds that $N_{D_{2}}^{-}\left(v_{1}\right)=\emptyset$ and $N_{D_{2}}^{-}\left(v_{2}\right) \subseteq\left\{v_{1}\right\}$. If $N_{D}^{-}\left(v_{2}\right)=\left\{v_{1}\right\}$, then let $D$ be the digraph obtained from $D_{2}$ by deleting the $\operatorname{arc}\left(v_{1}, v_{2}\right)$. Otherwise, let $D=D_{2}$. Then $D$ is an acyclic digraph satisfying (a), (b), and (c). Thus the statement holds.

The competition number of a cocktail party graph is given by Kim, Park and Sano [6].

Proposition 2.3 ([6]) The competition number of a cocktail party graph $C P(m)$ with $m \geq 2$ is equal to two.

\subsection{Proof of Theorem 1.2}

In this subsection, we show that the competition number of a generalized line graph is at most two.

For two vertex-disjoint graphs $G$ and $H$ and a clique $K$ of $G$, we define the graph $G \ltimes_{K} H$ by

$$
\begin{aligned}
& V\left(G \ltimes_{K} H\right):=V(G) \cup V(H) \\
& E\left(G \ltimes_{K} H\right):=E(G) \cup E(H) \cup\{u v \mid u \in K, v \in V(H)\}
\end{aligned}
$$

(see Figure 2).

From Lemma 2.2, we introduce a notion of top-two of a graph as follows.

Definition 1 For a graph $G$, a set $\{u, v\}$ of two distinct vertices $u$ and $v$ of $G$ is called a top-two of $G$ if there exists an acyclic digraph $D$ such that $C(D)=G \cup I_{k(G)}$ and $D$ has an acyclic ordering whose first and second vertices are $u$ and $v$.

Note that any graph with at least two vertices always has at least one top-two. If a graph $G$ has no edges, then any pair of two vertices of $G$ is a top-two of $G$.

Proposition 2.4 Let $G$ and $H$ be graphs with at least two vertices such that $V(G) \cap V(H)=\emptyset$ and let $K$ be a clique of $G$. Suppose that there exists an acyclic digraph $D^{\prime}$ such that $C\left(D^{\prime}\right)=G \cup\left\{u_{1}, u_{2}\right\}$ where $\left\{u_{1}, u_{2}\right\}$ is a top-two of $H$. If either $H$ has no edges or $H$ has no isolated vertices, then there exists an acyclic digraph $D$ satisfying the following: 
(i) $C(D)=\left\{\begin{array}{ll}\left(G \ltimes_{K} H\right) \cup I_{2} & \text { if } H \text { has no edges, } \\ \left(G \ltimes_{K} H\right) \cup I_{|k(H)|} & \text { if } H \text { has no isolated vertices, }\end{array}\right.$ and

(ii) D has an acyclic ordering whose first $|V(G)|+2$ terms induce the digraph $D^{\prime}$.

Proof: Suppose that $H$ has no edges. Let $V(H)=\left\{u_{1}, u_{2}, \ldots, u_{m}\right\}$, where $m \geq 2$. We define a digraph $D$ by $V(D):=V(G) \cup V(H) \cup\left\{u_{m+1}, u_{m+2}\right\}$ and

$$
A(D):=A\left(D^{\prime}\right) \cup\left(\bigcup_{i=1}^{m}\left\{\left(x, u_{i+2}\right) \mid x \in K \cup\left\{u_{i}\right\}\right\}\right)
$$

where $u_{m+1}$ and $u_{m+2}$ are new vertices. Then, it is easy to see that $D$ is an acyclic digraph satisfying (i) and (ii).

Suppose that $H$ has no isolated vertices. Let $k:=k(H)$. By the assumption that $\left\{u_{1}, u_{2}\right\}$ is a top-two of $H$, there exists an acyclic digraph $D^{\prime \prime}$ such that $C\left(D^{\prime \prime}\right)=H \cup I_{k}$ and the two vertices $u_{1}$ and $u_{2}$ in $D^{\prime \prime}$ satisfy $N_{D^{\prime \prime}}^{-}\left(u_{1}\right)=N_{D^{\prime \prime}}^{-}\left(u_{2}\right)=\emptyset$. Let $D$ be the digraph defined by $V(D):=V\left(D^{\prime}\right) \cup V\left(D^{\prime \prime}\right)$ and $A(D):=A\left(D^{\prime}\right) \cup A\left(D^{\prime \prime}\right) \cup A^{*}$ where

$$
A^{*}:=\left\{(u, v) \mid u \in K, v \in V\left(D^{\prime \prime}\right) \backslash\left\{u_{1}, u_{2}\right\}\right\} .
$$

Since the ordering obtained by attaching an acyclic ordering of $D^{\prime \prime}$ at the end of an acyclic ordering of $D^{\prime}$ gives an acyclic ordering of $D, D$ is acyclic and satisfies (ii). Since $H$ has no isolated vertices, each vertex in $H$ has an out-neighbor in $D^{\prime \prime}$. Therefore, each edge of $G \ltimes_{K} H$ between a vertex in $K$ and a vertex of $H$ is an edge of $C(D)$ which results from $A^{*}$. Thus $C(D)=\left(G \ltimes_{K} H\right) \cup I_{k}$ and so $D$ satisfies (i).

For any vertex $v$ of a graph $H$, let $K_{H}(v)$ denote the set of the edges incident to $v$ in $H$. Note that $K_{H}(v)$ forms a clique of the line graph of $H$ for each vertex $v$ in a graph $H$ and $\left\{K_{H}(v) \mid v \in V(H)\right\}$ is an edge clique cover of the line graph of $H$.

Theorem 2.5 Let $(H, f)$ be a vertex-weighted graph. For any edge e $=u v$ of $H$, there exists an acyclic digraph $D$ such that $C(D)=L(H, f) \cup I_{2}$ and that $N_{D}^{-}\left(z_{1}\right)=K_{H}(u)$ and $N_{D}^{-}\left(z_{2}\right)=K_{H}(v)$, where $V\left(I_{2}\right)=\left\{z_{1}, z_{2}\right\}$.

Proof: Let $G:=L(H, f)$ for convenience. First, we consider the case where $f$ is a zero function. We show the theorem by induction on the number of edges of $H$. If $H$ has at most one edge, then the statement is checked easily. Assume that the statement is true for any graph with $m-1$ edges where $m \geq 2$. Let $H$ be a graph with $m$ edges. It is sufficient to consider the case where $H$ is connected. Take an edge $e=u v$ of $H$. Since $m \geq 2$ and $H$ is connected, there exists an edge $e^{\prime}$ incident to $e$ in $H$. Without loss of generality, we may assume that the vertex $u$ is also an endvertex of $e^{\prime}$. Let $H^{\prime}$ be the graph obtained from $H$ by deleting the edge $e$. Then $L\left(H^{\prime}\right)$ is the graph obtained from $L(H)$ by deleting the vertex $e$. Since $H^{\prime}$ has $m-1$ edges, by the induction hypothesis, there exists an acyclic digraph $D^{\prime}$ such that $C\left(D^{\prime}\right)=L\left(H^{\prime}\right) \cup\left\{z_{1}, e\right\}$ and $N_{D^{\prime}}^{-}\left(z_{1}\right)=K_{H^{\prime}}(u)$.

Now we define a digraph $D$ by $V(D):=V\left(D^{\prime}\right) \cup\left\{z_{2}\right\}=V(L(H)) \cup\left\{z_{1}, z_{2}\right\}$ and

$$
A(D):=A\left(D^{\prime}\right) \cup\left\{\left(e, z_{1}\right)\right\} \cup\left\{\left(e^{\prime \prime}, z_{2}\right) \mid e^{\prime \prime} \in K_{H}(v)\right\} .
$$


Then the ordering of the vertices in $D$ obtained from an acyclic ordering of $D^{\prime}$ by adding the vertex $z_{2}$ to it as the last term is an acyclic ordering of $D$, and so $D$ is acyclic. By the definitions of $D$ and $H^{\prime}$, $N_{D}^{-}\left(z_{2}\right)=K_{H}(v)$ and $N_{D}^{-}\left(z_{1}\right)=N_{D^{\prime}}^{-}\left(z_{1}\right) \cup\{e\}=K_{H^{\prime}}(u) \cup\{e\}=K_{H}(u)$. It is easy to see that $C(D)=L(H) \cup\left\{z_{1}, z_{2}\right\}$. Thus the theorem holds when $f$ is a zero function.

Second, we consider the case where $f$ is not a zero function. Let $v_{1}, v_{2}, \ldots, v_{n}$ be the vertices of $H$ such that $f\left(v_{i}\right)>0$. For each $i \in\{0\} \cup[n]$, we define a graph $G_{i}$ by

$$
G_{0}:=L(H) \quad \text { and } \quad G_{i}:=G_{i-1} \ltimes_{K_{H}\left(v_{i}\right)} Q_{v_{i}} \quad(i \in[n]),
$$

where $Q_{v_{i}}=C P\left(f\left(v_{i}\right)\right)$. Note that $G_{n}=G$.

Take an edge $e=u v$ of $H$. Since $f\left(v_{1}\right) \neq 0, Q_{v_{1}}$ has at least two vertices. Then $Q_{v_{1}}$ has a top-two $\left\{z_{1}, z_{2}\right\}$. Since $G_{0}$ is a line graph, by the above argument on the case of $f=0$, there exists an acyclic digraph $D_{0}$ such that $C\left(D_{0}\right)=G_{0} \cup\left\{z_{1}, z_{2}\right\}$ and $N_{D_{0}}^{-}\left(z_{1}\right)=K_{H}(u)$ and $N_{D_{0}}^{-}\left(z_{2}\right)=K_{H}(v)$.

Since $Q_{v_{1}}$ has no edges or $Q_{v_{1}}$ is connected, it follows from Propositions 2.3 and 2.4 that there exists an acyclic digraph $D_{1}$ such that $C\left(D_{1}\right)=G_{1} \cup I_{2}$ and $D_{1}$ has an acyclic ordering whose first $\left|V\left(G_{0}\right)\right|+2$ terms induce the digraph $D_{0}$. Then $N_{D_{1}}^{-}\left(z_{1}\right)=N_{D_{0}}^{-}\left(z_{1}\right)=K_{H}(u)$ and $N_{D_{1}}^{-}\left(z_{2}\right)=N_{D_{0}}^{-}\left(z_{2}\right)=K_{H}(v)$. From repeating the process, we can obtain an acyclic digraph $D_{n}$ such that $C\left(D_{n}\right)=G_{n} \cup I_{2}$ and $N_{D_{n}}^{-}\left(z_{1}\right)=K_{H}(u)$ and $N_{D_{n}}^{-}\left(z_{2}\right)=K_{H}(v)$. Let $D:=D_{n}$. Since $G_{n}=G$, the theorem holds.

Proof of Theorem 1.2: It immediately follows from Theorem 2.5 that the competition number of a generalized line graph is at most two.

\subsection{Generalized line graphs with competition number one}

In the following, we show some necessary conditions and sufficient conditions for the competition number of a connected generalized line graph being one. Theorem 1.1 says that the competition number of a connected line graph is one if and only if it has a simplicial vertex.

Since the case of the generalized line graph $L(H, f)$ of a vertex-weighted graph $(H, f)$ with a zero function $f$ is reduced to Theorem 1.1, we consider the case where $f$ is a nonzero function. In this subsection, when we consider the generalized line graph $L(H, f)$ of a vertex-weighted graph $(H, f)$, we denote the cocktail party graph $C P(f(v))$ added to $L(H)$ by $Q_{v}$ for each vertex $v$ of $H$ in cases where this notation will not cause confusion.

First, we give necessary conditions for the competition number of a connected generalized line graph being one.

Lemma 2.6 If a graph $G$ has competition number one, then $G$ has a simplicial vertex or an isolated vertex.

Proof: If $G$ has no simplicial vertex and no isolated vertex, then the competition number of $G$ is at least two by Proposition 2.1. Thus the lemma holds.

Theorem 2.7 Let $G$ be the generalized line graph of a connected vertex-weighted graph $(H, f)$ with a nonzero function $f$. If $k(G)=1$, then at least one of the following holds:

(i) $f(v)=1$ for some vertex $v$ of $H$, 


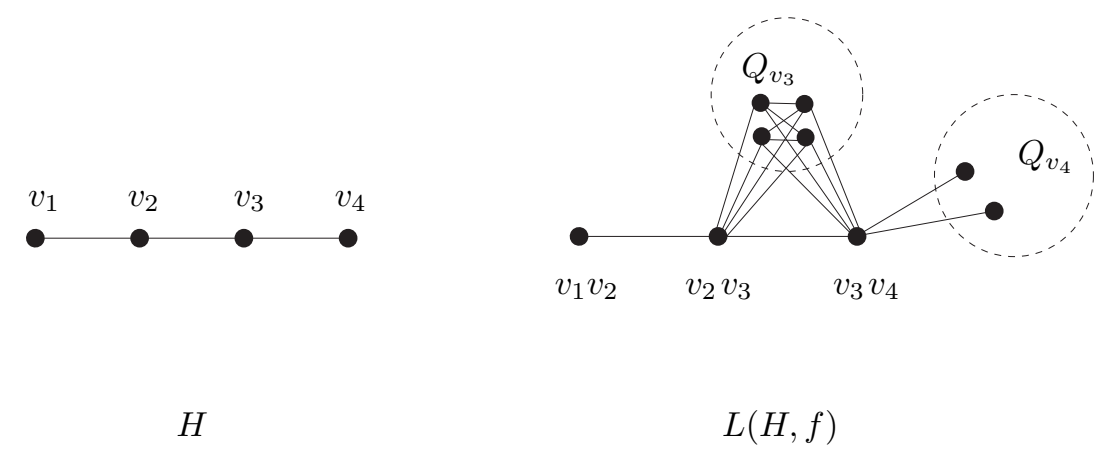

Fig. 3: A vertex-weighted graph $(H, f)$, where $f: V(H) \rightarrow \mathbb{Z}_{\geq 0}$ is defined by $f\left(v_{1}\right)=f\left(v_{2}\right)=0, f\left(v_{3}\right)=2$, $f\left(v_{4}\right)=1$, and its generalized line graph $L(H, f)$

(ii) There exists a vertex $v$ of $H$ such that $f(v)=0$ and $K_{H}(v)$ contains a simplicial vertex of $G$.

Proof: Assume that $k(G)=1$. Suppose that (i) does not hold. Then $f(u) \neq 1$ for any vertex $u \in$ $V(H)$. As $k(G)=1$ and $G$ is connected, $G$ has a simplicial vertex $x$ by Lemma 2.6. Since $V(G)=$ $\bigcup_{u \in V(H)}\left(K_{H}(u) \cup Q_{u}\right)$, the simplicial vertex $x$ is contained in $K_{H}(v) \cup Q_{v}$ for some $v \in V(H)$. Since $f(v) \neq 1$, either $f(v)=0$ or $f(v) \geq 2$. If $f(v) \geq 2$, then any vertex in $K_{H}(v) \cup Q_{v}$ is not simplicial, which is a contradiction. Therefore $f(v)=0$. Thus the simplicial vertex $x$ is contained in $K_{H}(v)$. Hence (ii) holds.

Remark 2.8 The conditions (i) and (ii) in Theorem 2.7 are not sufficient conditions for generalized line graphs to have competition number one.

Example 2.9 Let $(H, f)$ be the vertex-weighted graph where $H$ is the graph defined by $V(H)=\left\{v_{1}, v_{2}\right.$, $\left.v_{3}, v_{4}\right\}$ and $E(H)=\left\{v_{1} v_{2}, v_{2} v_{3}, v_{3} v_{4}\right\}$ and $f: V(H) \rightarrow \mathbb{Z}_{\geq 0}$ is defined by $f\left(v_{1}\right)=f\left(v_{2}\right)=0$, $f\left(v_{3}\right)=1, f\left(v_{4}\right)=2$ (see Figure 3). Then, the generalized line graph of $(H, f)$ satisfies both (i) and (ii) of Theorem 2.7. But, the competition number of $L(H, f)$ is two.

To see this, we recall a result by Kim [3] which states that the deletion of some pendant vertices from a connected graph does not change its competition number if the resulting graph has at least two vertices. Let $G^{\prime}$ be the graph obtained from $L(H, f)$ by deleting the two vertices in $Q_{v_{4}}$ and the vertex $v_{1} v_{2}$. Then, $k\left(G^{\prime}\right)=k(L(H, f))$. Since $G^{\prime}$ contains neither an isolated vertex nor a simplicial vertex, $k\left(G^{\prime}\right) \geq 2$ by Lemma 2.6. By Theorem 1.2, $k(L(H, f))=2$.

Next, we show the following result which gives sufficient conditions for generalized line graphs to have competition number one.

Theorem 2.10 Let $G$ be the generalized line graph of a connected vertex-weighted graph $(H, f)$ where $H$ has at least one edge and $f$ is a nonzero function. Then, $k(G)=1$ if one of the following holds:

(i) $f(u)=f(v)=1$ for some edge e $=$ uv of $H$,

(ii) $f(v) \leq 1$ for any vertex $v$ of $H$. 
Proof: Note that $G$ is a connected graph with at least two vertices, since $H$ is a connected graph with at least one edge and $f$ is a nonzero function. Therefore $k(G) \geq 1$. It is sufficient to show that there exists an acyclic digraph $D$ such that $C(D)=G \cup I_{1}$.

Suppose that (i) holds. Then, $f(u)=f(v)=1$ for some edge $u v$ of $H$ and therefore both $Q_{u}$ and $Q_{v}$ are the edgeless graph $I_{2}$ on two vertices. Let $V\left(Q_{u}\right)=\left\{q_{u}, q_{u}^{\prime}\right\}$ and $V\left(Q_{v}\right)=\left\{q_{v}, q_{v}^{\prime}\right\}$. Let $f_{0}: V(H) \rightarrow \mathbb{Z}_{\geq 0}$ be the function defined by $f_{0}(u)=f_{0}(v)=0$ and $f_{0}(x)=f(x)$ for any $x \in V(H) \backslash\{u, v\}$. Let $G_{0}$ be the generalized line graph of $\left(H, f_{0}\right)$. Then $G_{0}$ is isomorphic to the graph obtained from $G$ by deleting the four vertices $q_{u}, q_{u}^{\prime}, q_{v}$, and $q_{v}^{\prime}$. By Theorem 2.5, $k\left(G_{0}\right) \leq 2$ and there exists an acyclic digraph $D_{0}$ such that $C\left(D_{0}\right)=G_{0} \cup\left\{q_{v}, z\right\}$, where $z$ is a new vertex, and $K_{H}(u)=N_{D_{0}}^{-}\left(q_{v}\right)$ and $K_{H}(v)=N_{D_{0}}^{-}(z)$. Let $D$ be a digraph defined by $V(D):=V(G) \cup\{z\}$ and

$$
\begin{aligned}
A(D):= & A\left(D_{0}\right) \cup\left\{\left(q_{u}, q_{u}^{\prime}\right),\left(q_{u}^{\prime}, q_{v}\right),\left(q_{v}, q_{v}^{\prime}\right),\left(q_{v}^{\prime}, z\right)\right\} \\
& \cup\left\{\left(x, q_{u}^{\prime}\right) \mid x \in K_{H}(u)\right\} \cup\left\{\left(x, q_{v}^{\prime}\right) \mid x \in K_{H}(v)\right\} .
\end{aligned}
$$

Then $D$ is acyclic and $C(D)=G \cup\{z\}$. Therefore $k(G) \leq 1$.

Next, suppose that (ii) holds. Then $f(v) \leq 1$ for any vertex $v$ of $H$. Let $S:=\{v \in V(H) \mid f(v)=1\}$. Since $f$ is not a zero function, the set $S$ is not empty. Let $S:=\left\{u_{1}, u_{2}, \ldots, u_{t}\right\}$, where $t:=|S|>0$. Then $Q_{u_{i}}=C P(1)=I_{2}$ and let $V\left(Q_{u_{i}}\right)=\left\{q_{2 i-1}, q_{2 i}\right\}$ for $i \in[t]$. By Theorem 2.5, there exists an acyclic digraph $D_{0}$ such that $C\left(D_{0}\right)=L(H) \cup\left\{z, q_{2 t}\right\}$ where $z$ is a new vertex and $N_{D_{0}}^{-}(z)=K_{H}\left(u_{1}\right)$. Let $v_{1}, v_{2}, \ldots, v_{n}, q_{2 t}, z$ be an acyclic ordering of $D_{0}$. If $t=1$ then let $R:=\emptyset$, and if $t>1$ then let

$$
R:=\bigcup_{i=1}^{t-1}\left\{\left(x, q_{2 i}\right) \mid x \in K_{H}\left(u_{i+1}\right) \cup\left\{q_{2 i+1}\right\}\right\} .
$$

Let $D$ be the digraph defined by $V(D):=V\left(D_{0}\right) \cup\left\{q_{1}, \ldots, q_{2 t-1}\right\}=V(G) \cup\{z\}$ and

$$
A(D):=A\left(D_{0}\right) \cup\left\{\left(q_{1}, z\right)\right\} \cup\left(\bigcup_{i=1}^{t}\left\{\left(x, q_{2 i-1}\right) \mid x \in K_{H}\left(u_{i}\right) \cup\left\{q_{2 i}\right\}\right\}\right) \cup R .
$$

Then $D$ is acyclic since the ordering $v_{1}, v_{2}, \ldots, v_{n}, q_{2 t}, q_{2 t-1}, q_{2}, q_{1}, z$ of the vertices of $D$ is an acyclic ordering of $D$. In addition, it follows from the definitions of $D$ and $G$ that $C(D)=G \cup\{z\}$. Therefore $k(G) \leq 1$.

Thus $k(G)=1$, and hence the theorem holds.

Remark 2.11 Each of the conditions (i) and (ii) in Theorem 2.10 is not necessary conditions for generalized line graphs to have competition number one.

Example 2.12 Let $(H, f)$ be the vertex-weighted graph where $H$ is the graph defined by $V(H)=$ $\left\{v_{1}, v_{2}, v_{3}, v_{4}\right\}$ and $E(H)=\left\{v_{1} v_{2}, v_{1} v_{3}, v_{1} v_{4}\right\}$ and $f: V(H) \rightarrow \mathbb{Z}_{\geq 0}$ is the function defined by $f\left(v_{1}\right)=f\left(v_{2}\right)=f\left(v_{3}\right)=0$ and $f\left(v_{4}\right)=2$ (see Figure 4). Then, the generalized line graph of $(H, f)$ has the competition number one but $(H, f)$ satisfies neither (i) nor (ii) of Theorem 2.10. 


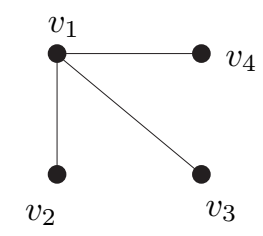

$H$

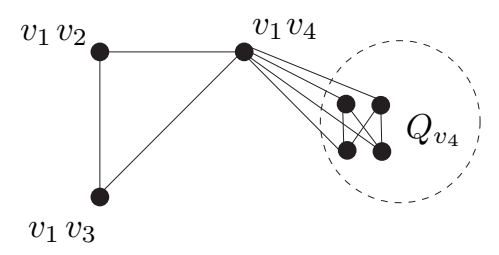

$L(H, f)$

Fig. 4: A vertex-weighted graph $(H, f)$, where $f: V(H) \rightarrow \mathbb{Z}_{\geq 0}$ is defined by $f\left(v_{1}\right)=f\left(v_{2}\right)=f\left(v_{3}\right)=0$ and $f\left(v_{4}\right)=2$, and its generalized line graph $L(H, f)$

Let $V\left(Q_{v_{4}}\right)=\left\{q_{1}, q_{2}, q_{3}, q_{4}\right\}$ and $E\left(Q_{v_{4}}\right)=\left\{q_{1} q_{2}, q_{2} q_{3}, q_{3} q_{4}, q_{4} q_{1}\right\}$. To see $k(L(H, f))=1$, we define a digraph $D$ by

$$
\begin{aligned}
V(D):= & V(L(H, f)) \cup\{z\}=E(H) \cup V\left(Q_{v_{4}}\right) \cup\{z\}, \\
A(D):= & \left\{\left(q_{1}, q_{2}\right),\left(q_{4}, q_{2}\right),\left(v_{1} v_{2}, q_{2}\right)\right\} \cup\left\{\left(q_{1}, q_{3}\right),\left(q_{2}, q_{3}\right),\left(v_{1} v_{2}, q_{3}\right)\right\} \\
& \cup\left\{\left(q_{2}, v_{1} v_{3}\right),\left(q_{3}, v_{1} v_{3}\right),\left(v_{1} v_{2}, v_{1} v_{3}\right)\right\} \cup\left\{\left(q_{3}, v_{1} v_{4}\right),\left(q_{4}, v_{1} v_{4}\right),\left(v_{1} v_{2}, v_{1} v_{4}\right)\right\} \\
& \cup\left\{\left(v_{1} v_{2}, z\right),\left(v_{1} v_{3}, z\right),\left(v_{1} v_{4}, z\right)\right\}
\end{aligned}
$$

where $z$ is a new vertex. Then we can easily check that $C(D)=L(H, f) \cup\{z\}$, and that $D$ is acyclic since the ordering $q_{1}, q_{4}, v_{1} v_{2}, q_{2}, q_{3}, v_{1} v_{3}, v_{1} v_{4}, z$ is an acyclic ordering of $D$. Therefore $k(L(H, f)) \leq 1$, Since $L(H, f)$ is connected, $k(L(H, f)) \geq 1$. Hence $k(L(H, f))=1$.

\section{Concluding Remark}

In this note, we showed that the competition number of a generalized line graph is at most two, which is an extension of a result on the competition number of a line graph. In addition, we tried to characterize generalized line graphs whose competition numbers are one, and then found necessary conditions and sufficient conditions for the competition number of a generalized line graph being one. However, these conditions are not necessary-and-sufficient conditions, so it still remains open to give a complete characterization of generalized line graphs whose competition numbers are one.

\section{Acknowledgements}

The authors are grateful to anonymous referees for their careful reading and helpful comments. 


\section{References}

[1] J. E. Cohen: Interval graphs and food webs: a finding and a problem, Document 17696-PR, RAND Corporation, Santa Monica, CA (1968).

[2] A. J. Hoffman: $-1-\sqrt{2}$, Combinatorial structures and their applications (Proc. Calgary International Conf. Combin. structures and their appl., June 1969) (Eds. R. Guy et al.), Gordon and Breach, New York, (1970) 173-176.

[3] S. -R. Kim: Graphs with one hole and competition number one, Journal of the Korean Mathematical Society 42 (2005) 1251-1264.

[4] S. -R. Kim and Y. Sano: The competition numbers of complete tripartite graphs, Discrete Applied Mathematics 156 (2008) 3522-3524.

[5] S. -R. Kim, B. Park, and Y. Sano: The competition numbers of Johnson graphs, Discussiones Mathematicae Graph Theory 30 (2010) 449-459.

[6] S. -R. Kim, B. Park, and Y. Sano: The competition numbers of complete multipartite graphs with many partite sets, Discrete Applied Mathematics 160 (2012) 1176-1182.

[7] S. -R. Kim and F. S. Roberts: Competition numbers of graphs with a small number of triangles, Discrete Applied Mathematics 78 (1997) 153-162.

[8] R. J. Opsut: On the computation of the competition number of a graph, SIAM Journal on Algebraic and Discrete Methods 3 (1982) 420-428.

[9] B. Park, S. -R. Kim, and Y. Sano: The competition numbers of complete multipartite graphs and mutually orthogonal Latin squares, Discrete Mathematics 309 (2009) 6464-6469.

[10] B. Park and Y. Sano: The competition numbers of Hamming graphs with diameter at most three, Journal of the Korean Mathematical Society 48 (2011) 691-702.

[11] B. Park and Y. Sano: The competition numbers of ternary Hamming graphs, Applied Mathematics Letters 24 (2011) 1608-1613.

[12] F. S. Roberts: Food webs, competition graphs, and the boxicity of ecological phase space, Theory and applications of graphs (Proc. Internat. Conf., Western Mich. Univ., Kalamazoo, Mich., 1976) Lecture Notes in Mathematics 642, Springer (1978) 477-490.

[13] Y. Sano: The competition numbers of regular polyhedra, Congressus Numerantium 198 (2009) 211219.

[14] Y. Sano: A generalization of Opsut's lower bounds for the competition number of a graph, Graphs and Combinatorics, Published online (2012). doi : 10.1007/s00373-012-1188-5 